$1 \quad$ Streptococcus equi subsp. zooepidemicus associated with sudden death of swine in North

2 America

3

$4 \quad$ Running Title: Streptococcus equi subsp zooepidemicus in pigs

5 Keywords: Streptococcus zooepidemicus, sudden death, pigs, lymphnode, mortality,

6 zooepidemicus, outbreak, north America, pneumonia.

8 Authors: Matheus de O. Costa ${ }^{1,2}$, Brad Lage $^{3}$.

9 Affiliations:

$10{ }^{1}$ University of Minnesota, Department of Veterinary Population Medicine, Saint Paul, MN. USA.

11 (M. O. Costa).

$12{ }^{2}$ University of Saskatchewan, Large Animal Clinical Sciences. Saskatoon, SK. Canada. (M.O.

13 Costa)

$14{ }^{3}$ Maple Leaf Agri-Farms, Landmark Manibota, Canada. (B. Lage). 


\section{Abstract-50 words}

Historically described as a commensal of the swine upper-respiratory tract, Streptococcus equi subsp. zooepidemicus was only reported previously in Asia as an important swine pathogen.

Here we report the isolation and whole genome characterization of Streptococcus equi subsp. zooepidemicus associated with a sudden death outbreak in pigs in North America.

Text-764 words

Streptococcus equi subsp. zooepidemicus (S. zooepidemicus) is considered a commensal and opportunistic pathogen of several warm-blooded hosts, including humans, horses, different canines and swine. It is a Gram-positive, $\beta$-hemolytic coccus belonging to the Lancefield group C. It can cause severe disease characterized by pneumonia, septicemia and meningitis $(1,2) . S$. zooepidemicus has been suggested as a normal inhabitant of the palatine tonsils of pigs, being detected by both culture and high-throughput sequencing in samples collected from healthy animals (3). However, strains virulent to pigs have also been reported in the literature,

34 particularly associated with high-mortality outbreaks of sudden death and respiratory disease in China (4). Currently, there are no vaccines available for this pathogen and control and prevention methods are hardly applied, given its commensal nature in swine. Here, we report an outbreak of

37 sudden death associated with $S$. zooepidemicus in pigs housed in intensive rearing, commercial facilities in North America.

In April 2019 an outbreak of sudden death and abortions occurred in 4 loose-housed, commercial sow farms (approximately 9000 sows) in a large vertically integrated swine system 
42 affected sows herds by more than 1000 sows in the following 12 weeks. The abortion rate during

43 this time period was approximately $11 \mathrm{x}$ the normal rate. Animals were often described as

44 apparently healthy during morning checks. Over the course of hours, sows would become

45 unwilling to stand, develop fever, lethargy and die with no other apparent clinical signs. Other

46 sows would abort and then go on to develop similar symptoms. Stressing factors in these farms,

47 such as mixing of animals and the presence of other sick animals appeared to exacerbate

48 outbreaks within pens. Animals were fed a commercial grade, nutritionally balanced diet as per

49 ESF (electronic sow feeding) and had access to water ad libitum. Gross post-mortem

50 examination of multiple animals, either euthanized or recently deceased, revealed the following

51 common observations: rhinitis (mucopurulent discharge, mild, diffuse), pulmonary edema, gall

52 bladder edema, hemorrhagic lymphadenopathy (tan to haemorrhagic) consisting of

53 submandibular, cervical neck and bronchial lymph nodes, which taken together are suggestive of

54 sepsis. All animals tested negative for PRRSV, Mycoplasma hyopneumoniae, SIV-A, PCV-3 and

55 PCV-2 by real-time PCR. In parallel, Gram positive cocci were observed in imprints from heart

56 and submandibular lymph nodes. Aerobic bacterial culture followed by Matrix-Assisted Laser

57 Desorption/Ionization-Time Of Flight (MALDI-TOF) for identification of isolates revealed

58 varying levels of $S$. zooepidemicus in liver, kidney, heart, brain, lung, spleen, and submandibular

59 lymphnodes. Isolate identification was confirmed by two different veterinary diagnostic

laboratories. Isolates (n=7, SAMN13058951, SAMN13058952, SAMN13058953,

62 lincomycin, neomycin and tetracycline and susceptible to ampicillin, ceftiofur, penicillin and

63 tilmicosin in a Kirby-Bauer disk diffusion assay. 
DNA extraction was performed from isolates (DNeasy Powersoil Pro kit, Qiagen), quantified by Nanodrop (3300) and PicoGreen (Quant-iT dsDNA) and processed for sequencing paired-end). Samples yield an average of 149,017 high quality reads, suggesting 50x coverage (genome size averaged $2.1 \mathrm{mbp}$ ). Genome assembly, annotation and downstream analyses were conducted using the PATRIC package (5). Genomes averaged 2.1 million bp in size, and $41.34 \%$ in GC content. All isolates were similar to previously published S. zooepidemicus genomes (Figure 1), demonstrating a whole-genome average nucleotide identity (ANIscore) of $99.7 \%$ to strain S. zooepidemicus ATCC35246. This particular strain was reported as isolated from a septicaemic pig during an outbreak that killed over 300,000 pigs in Sichuan province, China, in 1976 (6). Interestingly, all isolates had an average ANIscore of 97.3\%, when compared to $S$. zooepidemicus strain 4047, an isolate considered virulent, obtained from a horse diagnosed with strangles in the United Kingdom (7). In addition, all isolates obtained from pigs, regardless of what outbreak, were profiled as MLST (multi-locus sequence type) ST-194, including strain ATCC35246. Antimicrobial resistance genes identified in isolates from this outbreak included gidB, $S 12 p$ (streptomycin), rpoB (rifampin), S10p (tetracycline), kasA (triclosan), PgsA, LiaR,

81 factors found included the previously described $s z m, \operatorname{lmb}, f b p Z, s k c$, has operon and $m a g$

82 regulon, which help explain the highly-virulent lifestyle of these isolates. cause of mortality in pigs in North America. This specific sequence type seem to be particularly 
here, this pathogen requires special attention and should no longer be overlooked, due to its

87 historically accepted commensal lifestyle, when conducting diagnostic investigations.

88

89

90

91

\section{Acknowledgments}

assistance.

The authors would like to acknowledge the Manitoba Veterinary Diagnostic Services for

Author Bio (first author only, unless there are only 2 authors)

interests include swine bacterial pathogens to which no preventive or control methods are available besides antibiotics. Dr. Lage is the head Veterinarian at Maple Leaf Agri-Farms, the swine division for Maple Leaf Foods based in Landmark, Manitoba.

\section{References}

1. Pelkonen S, Lindahl SB, Suomala P, Karhukorpi J, Vuorinen S, Koivula I, et al. Transmission of Streptococcus equi subspecies zooepidemicus infection from horses to humans. Emerg Infect Dis. 2013;19(7):1041-8. Epub 2013/06/20. doi: 10.3201/eid1907.121365. PubMed PMID: 23777752; PubMed Central PMCID: PMCPMC3713971.

2. FitzGerald W, Crowe B, Brennan P, Cassidy JP, Leahy M, McElroy MC, et al. Acute fatal haemorrhagic pneumonia caused by Streptococcus equi zooepidemicus in greyhounds in Ireland with subsequent typing of the isolates. Vet Rec. 2017;181(5):119. Epub 2017/06/11. doi: 10.1136/vr.104275. PubMed PMID: 28600445.

3. Kernaghan S, Bujold AR, MacInnes JI. The microbiome of the soft palate of swine. Anim Health Res Rev. 2012;13(1):110-20. Epub 2012/08/03. doi: 10.1017/S1466252312000102. PubMed PMID: 22853946.

4. Feng Z, Hu J. Outbreak of swine streptococcosis in Sichan province and identification of pathogen. Anim Husbandry Vet Med Lett. 1977;2:7-12.

5. Wattam AR, Davis JJ, Assaf R, Boisvert S, Brettin T, Bun C, et al. Improvements to PATRIC, the all-bacterial Bioinformatics Database and Analysis Resource Center. Nucleic Acids Res. 2017;45(D1):D535-D42. Epub 2016/12/03. doi: 10.1093/nar/gkw1017. PubMed PMID: 27899627; PubMed Central PMCID: PMCPMC5210524.

6. Waller AS, Robinson C. Streptococcus zooepidemicus and Streptococcus equi evolution: the role of CRISPRs. Biochem Soc Trans. 2013;41(6):1437-43. Epub 2013/11/22. doi: 10.1042/BST20130165. PubMed PMID: 24256234. 
117 7. Kelly C, Bugg M, Robinson C, Mitchell Z, Davis-Poynter N, Newton JR, et al. Sequence 118 variation of the SeM gene of Streptococcus equi allows discrimination of the source of strangles 119 outbreaks. J Clin Microbiol. 2006;44(2):480-6. Epub 2006/02/04. doi: 10.1128/JCM.44.2.480120 486.2006. PubMed PMID: 16455902; PubMed Central PMCID: PMCPMC1392674.

122 Figure 1. Phylogenetic tree (all-shared proteins) of Streptococcus equi subsp. zooepidemicus

123 whole-genome sequences obtained from the reported outbreak in pigs from North America (blue 124 blocks, PRJNA578379), compared with previously published human, dog, horse and pig (green 125 blocks) sequences from GenBank $(n=58)$. Tree inferred using BLAST followed by FastTree

126 within the PATRIC package(5). Support values shown indicate the number of times a particular 127 branch was observed in the support trees using gene-wise jackknifing. 
Streptococcus equi subsp. equi strain EQUI0059 $\longrightarrow$ Streptococcus equi subsp. equi strain EQUI0061 $\longrightarrow$ Streptococcus equi subsp. equil strain EQU10174 - Streptococcus equi subsp. equi strain EQUIO175

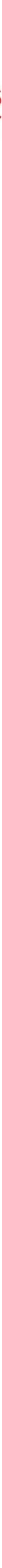

